\title{
Technology development roadmap for Habitable-zone exoplanet observatory (HabEx) baseline 4-m primary mirror
}

\author{
H. Philip Stahl \\ NASA \\ MSFC, AL 35812
}

Rhonda Morgan

Jet Propulsion Laboratory, California Institute of Technology

Pasadena, CA 91109 


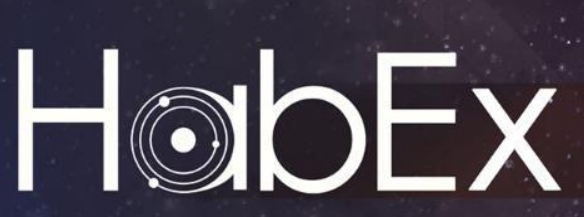

\section{Purpose of HabEx}

EXPLORING PLANETARY SYSTEMS AROUND NEARBY SUNLIKE STARS AND ENABLING OBSERVATORY SCIENCE FROM THE UV THROUGH NEAR-IR

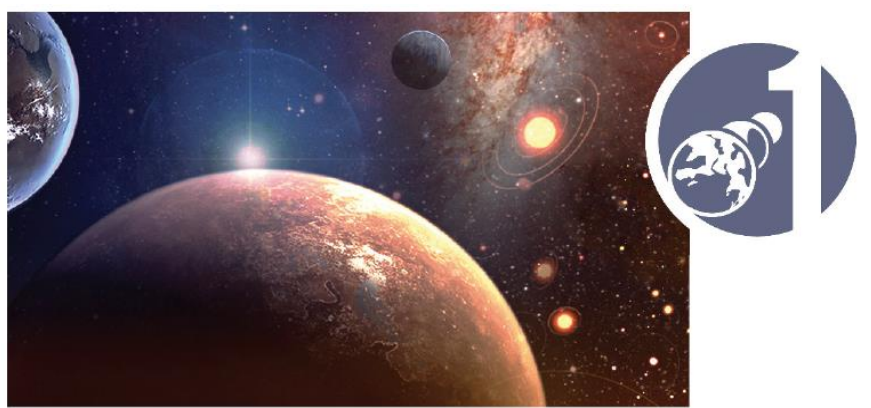

\section{GOAL 1}

To seek out nearby worlds and explore their habitability, HabEx will search for habitable zone Earth-like planets around sunlike stars using direct imaging and will spectrally characterize promising candidates for signs of habitability and life.

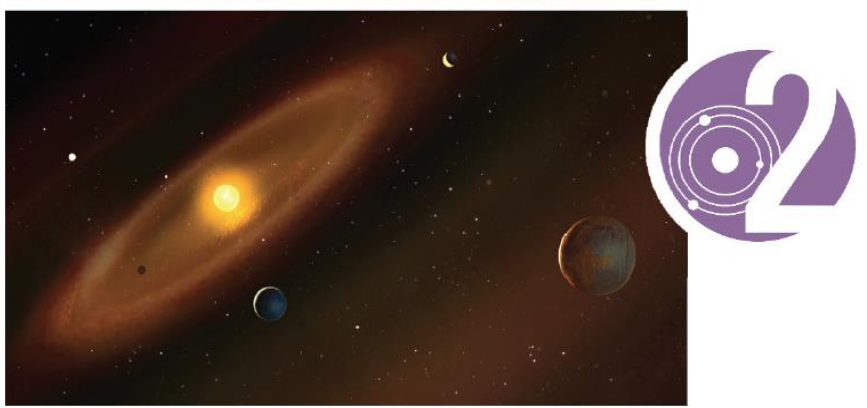

\section{GOAL 2}

To map out nearby planetary systems and understand the diversity of the worlds they contain, $\mathrm{HabEx}$ will take the first "family portraits" of nearby planetary systems, detecting and characterizing both inner and outer planets, as well as searching for dust and debris disks.

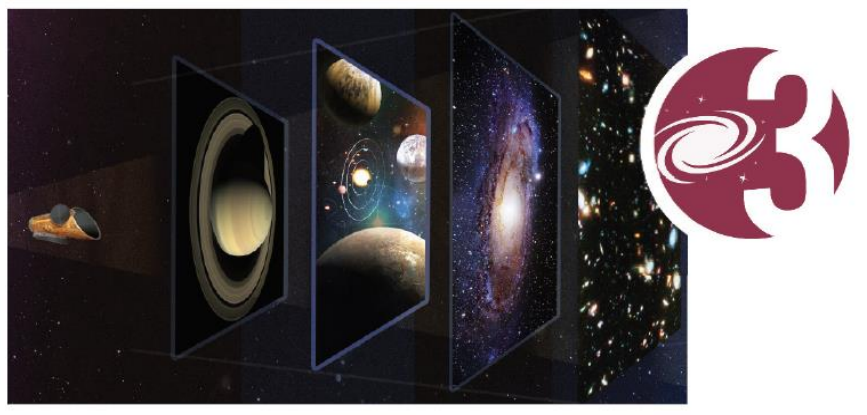

GOAL 3

To carry out observations that open up new windows on the universe from the UV through near-IR, HabEx will have a community driven, competed Guest Observer program to undertake revolutionary science with a large-aperture, ultra-stable UV through near-IR space telescope. 


\section{HobEx}

\section{Architecture A Concept}

The HabEx STDT chose these parameters for Architecture A:

Telescope with a $4 \mathrm{~m}$ aperture

52-m diameter, formation flying external Starshade occulter

Four instruments:

Coronagraph Instrument for Exoplanet Imaging

Starshade Instrument for Exoplanet Imaging

UV- Near-IR Imaging Multi-object Slit Spectrograph for General Science

High Resolution UV Spectrograph for General Observatory Science

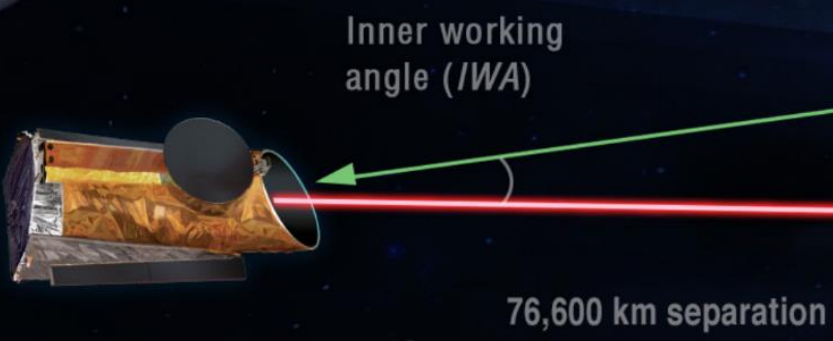




\section{HobEx}

\section{Báseline Design}

Baseline Observatory is Telescope surrounded by Spacecraft.

Only connection between two is Interface Ring.

Interface Ring is also where Observatory attaches to SLS PAF.

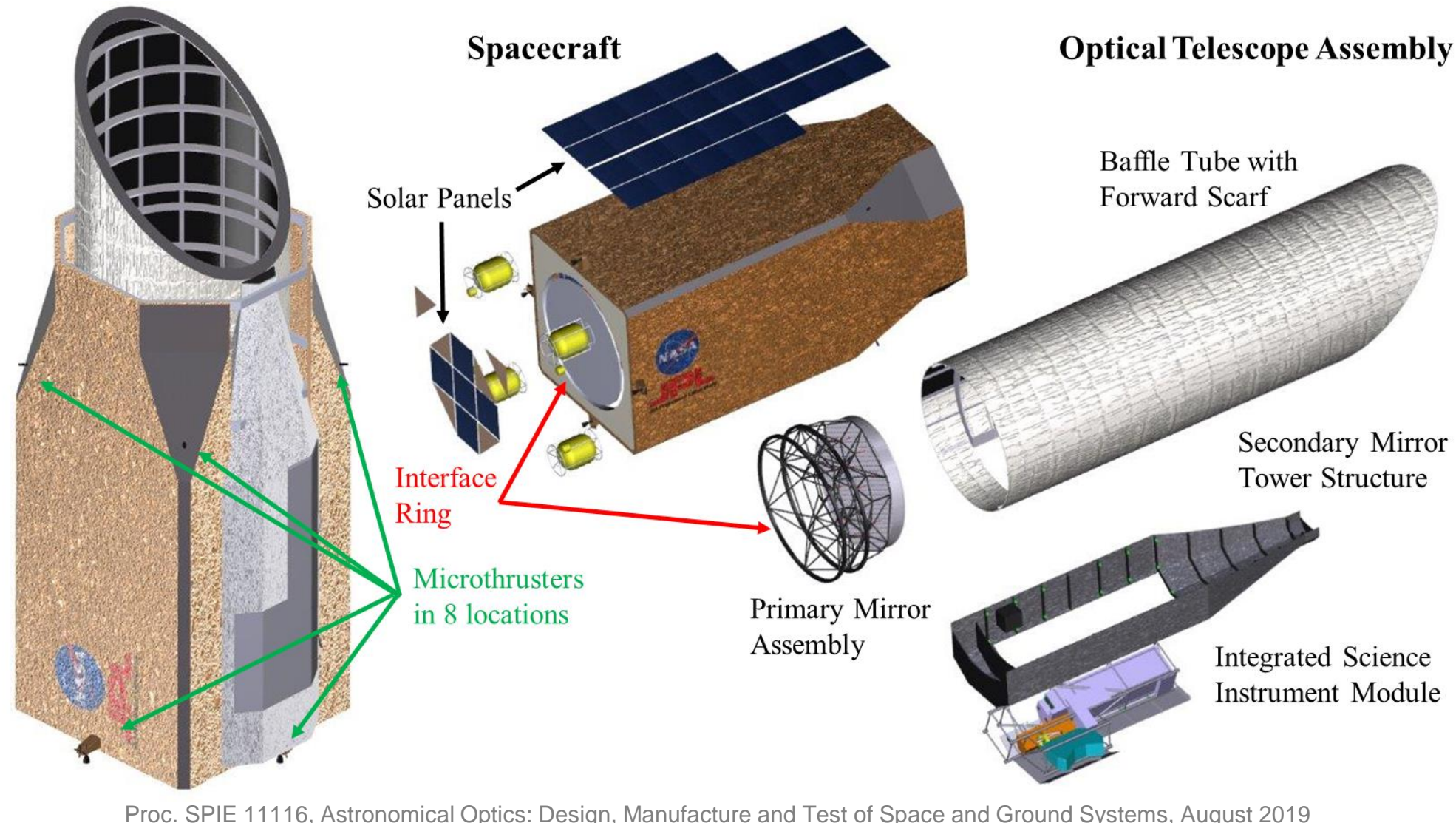


Architecture

Aperture Dia

LOS Stability

Diffraction Limit

Wavefront Error

Primary Mirror

WFE Stability
Unobscured Off-Axis F/2.5 TMA

4-meters Monolithic (Minimum)

$<2$ mas on-sky jitter (astrophysics and starshade)

$<0.7$ milli-arc-second on-sky jitter (coronagraph)

$400 \mathrm{~nm}$

30 nm rms Total

Total SFE $\quad<8 \mathrm{~nm}$ rms

$$
\begin{array}{ll}
\text { Low-Order }(<7 \mathrm{cpd}) & <7 \mathrm{~nm} \mathrm{rms} \\
\text { Mid-Spatial }(7 \text { to } 100 \mathrm{cpd}) & <6 \mathrm{~nm} \mathrm{rms} \\
\text { High-Spatial }(>100 \mathrm{cpd}) & <0.8 \mathrm{~nm} \mathrm{rms}
\end{array}
$$

< $5 \mathrm{~nm}$ rms (astrophysics and starshade)

$<1$ to $200 \mathrm{pm}$ rms per spatial frequency (coronagraph) 


\section{HobEx}

HabEx telescope optical design is off-axis TMA.

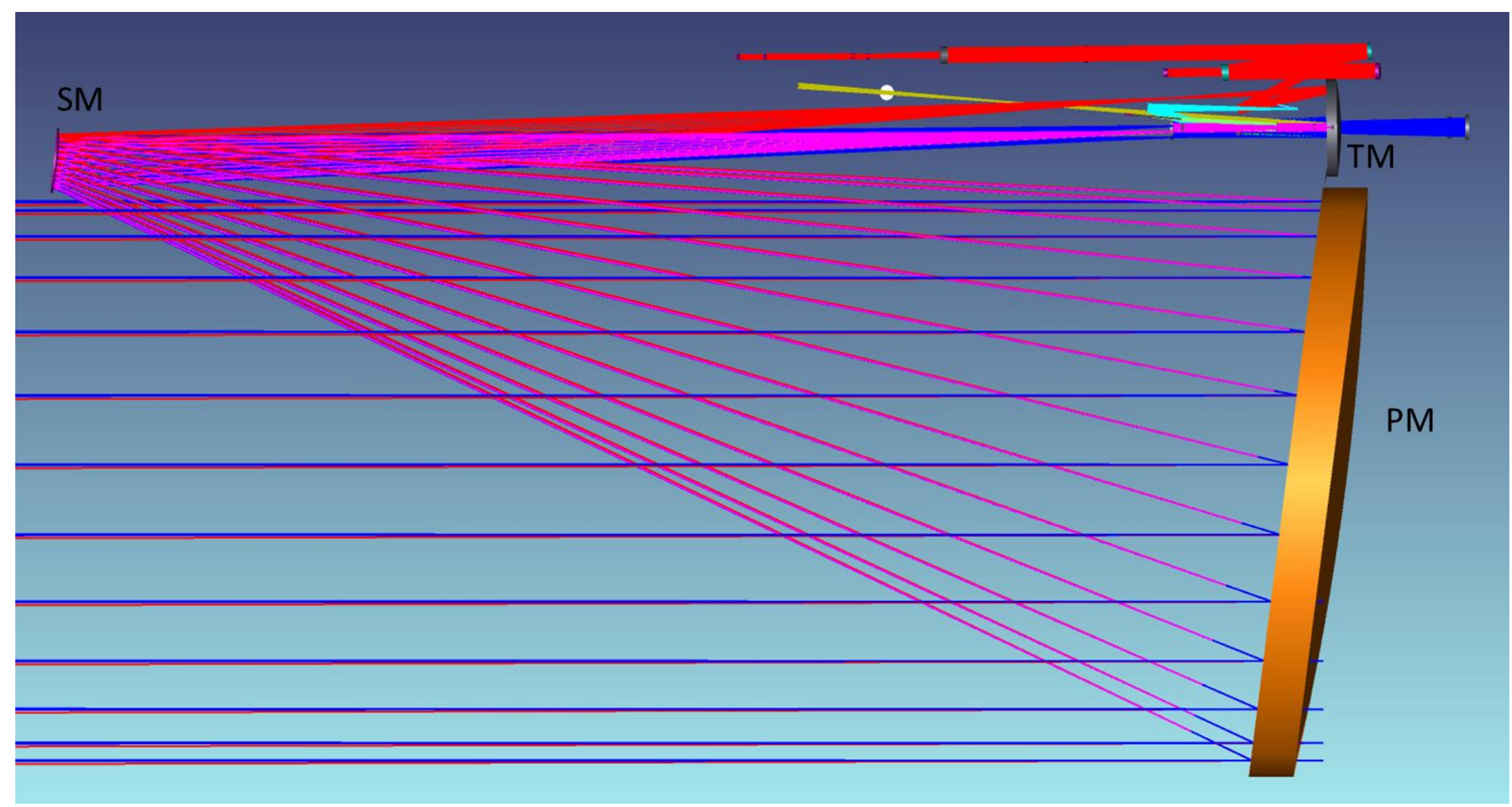




\section{HabEx Baseline Telescope}

\section{Science Driven Systems Engineering}




\section{‘The' System Challenge: Dark Hole}

Imaging an 'exo-Earth' requires blocking $10^{10}$ of host star's light. Internal coronagraph (with deformable mirrors) can create a 'dark hole' with $<10^{-10}$ contrast.
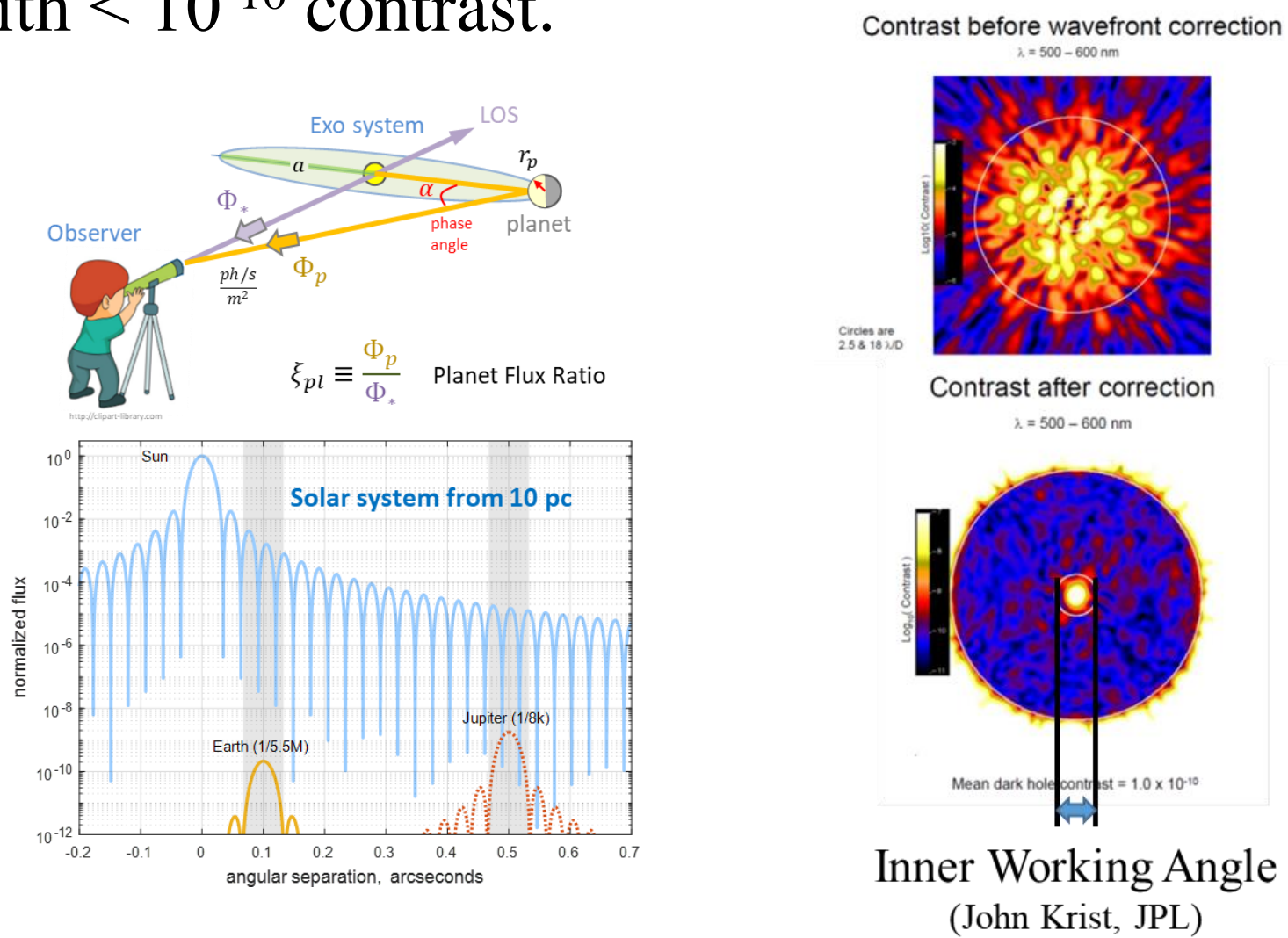

Krist, Trauger, Unwin and Traub, "End-to-end coronagraphic modeling including a low-order wavefront sensor", SPIE Vol. 8422, 844253, 2012; doi: 10.1117/12.927143 


\section{IWA and Core Throughput}

The greater the core throughput at the smallest possible IWA, the larger the number of habitable zones that can be searched.

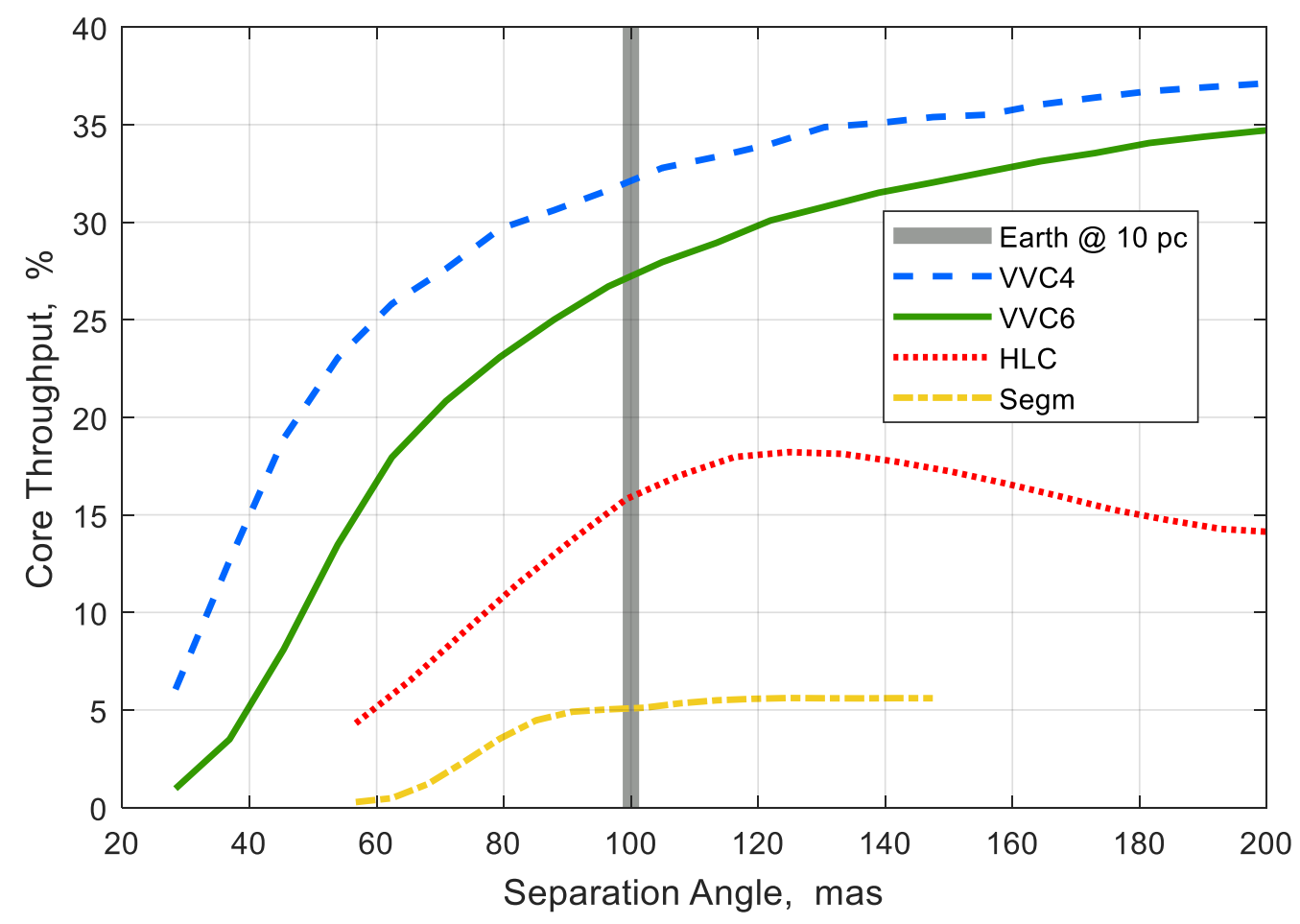

IWA is driven by

- PSF Size

- PSF Stability
Aperture Diameter \& Off-Axis Architecture LOS Jitter and WFE Stability Specification 


\section{Why Off-Axis}

Science depends on the telescope Point Spread Function (PSF) and the angular size of the 80\% Encircled Energy (EE) circle:

- Inner Working Angle (IWA) Expolanet Science

- Angular Resolution General Astrophysics

IWA is how close to a host star the coronagraph can detect an exo-planet - based on its ability to block light from the host star.

The more compact the PSF, the smaller the IWA.

PSF size depends on Telescope aperture diameter.

$\mathrm{PSF}$ central lobe angular radius $=1.22 \lambda / \mathrm{D}$.

$83 \%$ of the energy is in the central lobe.

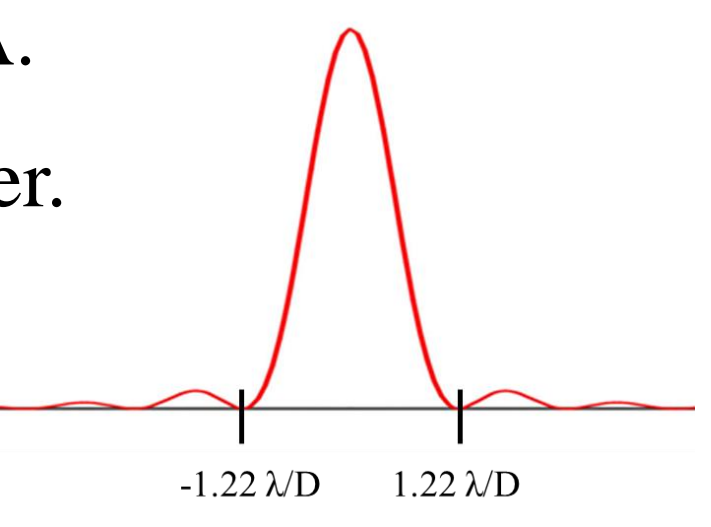

The larger the telescope aperture, the smaller the PSF and IWA. 


\section{Why Off-Axis}

\section{But, PSF is also affected by central obscuration and spiders.}

Diffraction from central obscuration and spiders broaden the PSF and move energy out of the central core.
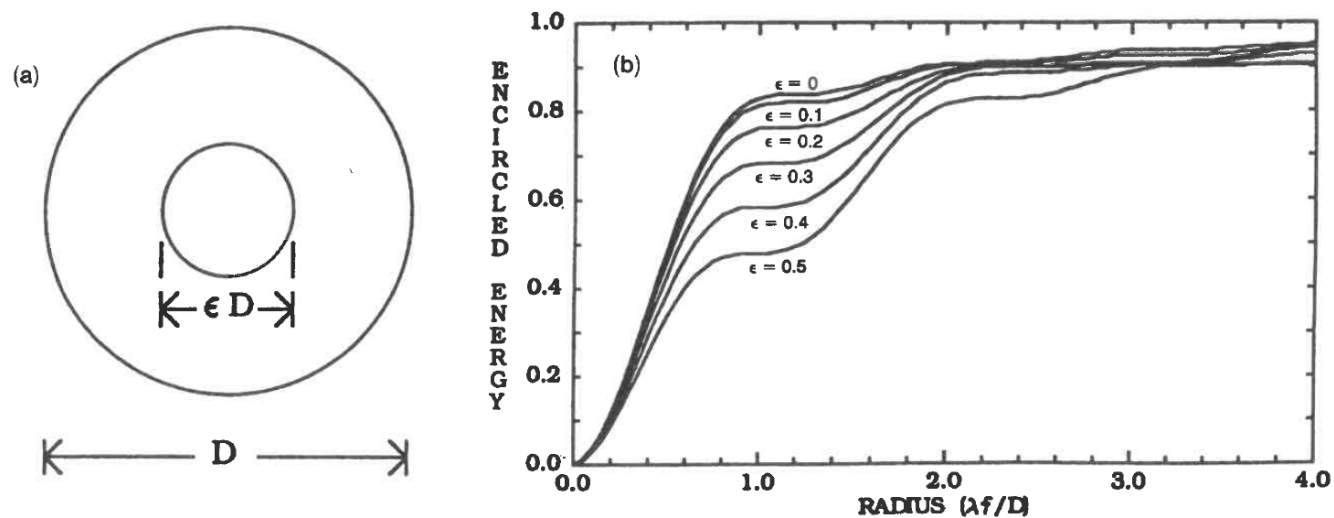

Fig. 6. Encircled energy caused by the diffraction-limited annular apertures. This figure can be used as a set of characteristic curve from which to obtain values of $E E_{\text {annulus }}(r)$, which are necessary when the empirical equation is applied to various aperture configurations.

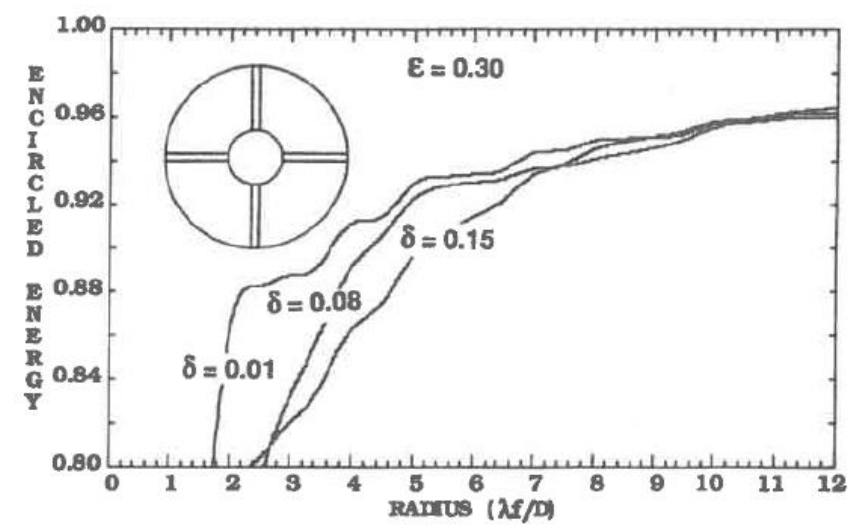

Fig. 13. Corresponding fractional encircled energy curves providing insight into the image-degradation effects of secondary mirror spiders of varying widths.

\section{Thus, an off-axis unobscured aperture has a smaller IWA than an on-axis centrally obscured aperture.}

Harvey, James E. and Christ Ftaclas, "Diffraction effects of telescope secondary mirror spiders on various image-quality criteria”, Applied Optics, Vol.34, No.28, p.6337, 1 Oct 1995. 


\section{Diffraction Limited Performance}

Diffraction limited performance (in addition to aperture) drives PSF size:

- General Astrophysics Resolution

- Coronagraphy Inner Working Angle

Diffraction limited performance is determined by transmitted wavefront error (WFE). 


\section{Primary Mirror Total Surface Figure Requirement}

Primary Mirror requirements are derived by flowing System Level diffraction limited and pointing stability requirements to major observatory elements:

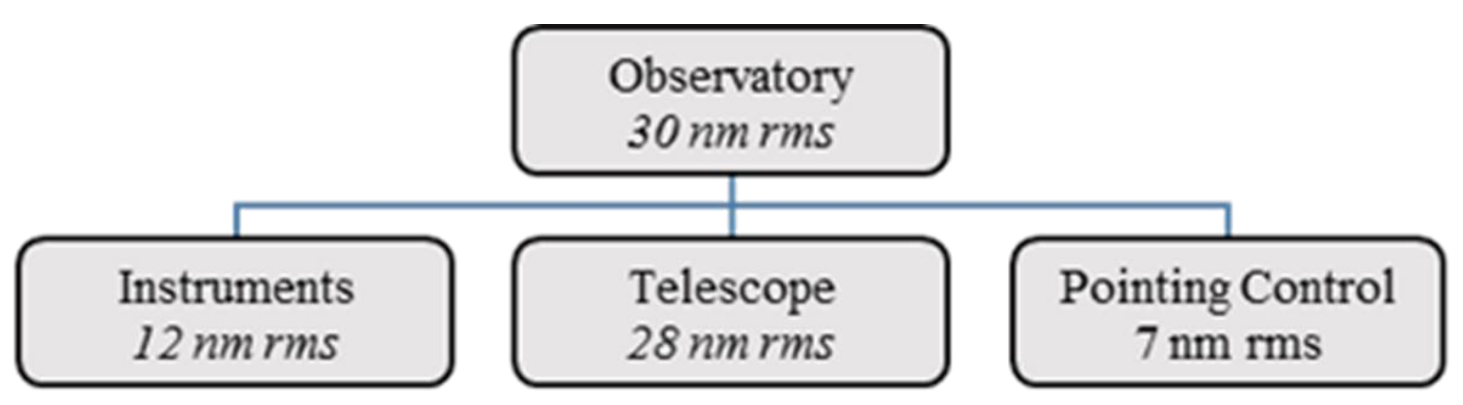

Then flowing Telescope Requirements to major Sub-Systems

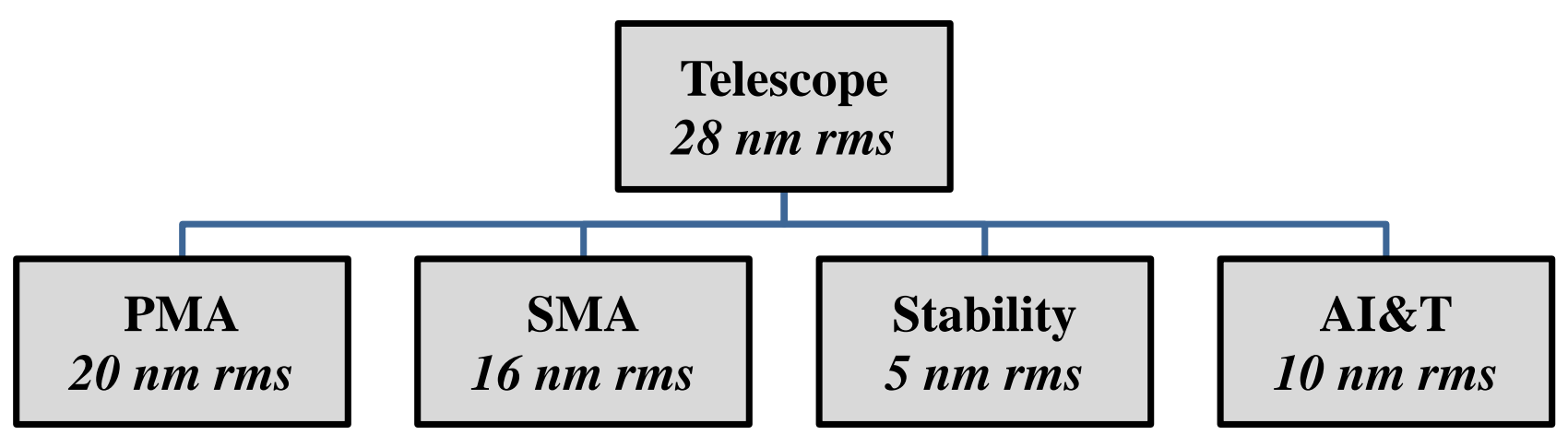




\section{Primary Mirror Total Surface Figure Requirement}

PM must have $<10 \mathrm{~nm}$ rms surface.

PM Specification depends on thermal behavior \& mounting uncertainty, leaving $<\sim 8 \mathrm{~nm}$ rms for total manufactured SFE.

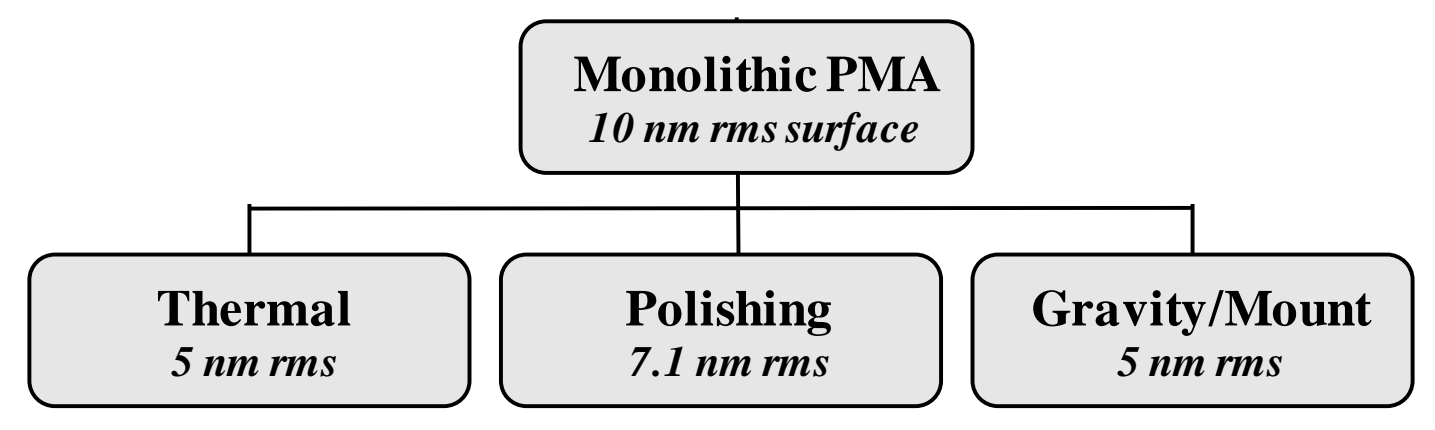

PM must be very smooth.

Mid-spatial frequency errors move light from core into 'hole'

DM moves that light back into the core.

High-spatial errors (3X OWA) 'fold' or 'scatter' light into 'hole'

Errors above DM range produce speckles whose amplitude varies as $1 / \lambda^{2}$ 


\section{Spatial Frequency vs Science}

Low spatial frequency specification is driven by General Astrophysics (not Exoplanet) science.

Exoplanet instruments have deformable mirrors to correct low-spatial errors and General Astrophysics instruments typically do not.

Mid/High spatial frequency specification is driven by Exoplanet because of 'leakage' or 'frequency folding'.

For exoplanet, the spatial band is from the inner working angle (IWA) to approximately $3 \mathrm{X}$ the outer working angle (OWA).

Theoretically, a 64 x $64 \mathrm{DM}$ can correct spatial frequencies up to 32 cycles per diameter (N/2), therefore, the maximum midspatial frequency of interest is $\sim 90$ cycles.

Since mirrors are smooth \& DM controllability rolls-off near N/2 limit, a conservative lower limit is $\sim N / 3$ or $\sim 20$ cycles. 


\section{Risks to Diffraction Limited Performance}

Assuming that mirrors are made to their prescription, biggest potential WFE source is ability to align mirrors and maintain that alignment on-orbit.

- Mitigate risk by designing hexapod actuators range.

- Mitigate risk by using laser position metrology system.

Another potentially error source is ability to quantify and back-out gravity effects.

Because mirrors are made in $1-\mathrm{G}$ and operated in $0-\mathrm{G}$ there is a shape difference - sometimes called G-release.

- Mitigate risk by active mirror control with actuator placement optimized to control most likely error modes. 


\section{Line of Sight (LOS) In-Stability}

LOS in-stability causes PSF smear and beam-shear WFE.

LOS in-stability has two causes:

- Jitter - response of structure to mechanical accelerations

- Drift - response of structure to changes in thermal environment

Specification of $<0.3$ mas rms per axis is uncorrectable Jitter and residual Drift after correction by Laser-truss system. 


\section{Wavefront In-Stability}

WFE Drift cause speckles which can produce a false exoplanet measurement or mask a true signal.

Spatial frequency of that error is important.

Three sources of WFE in-stability:

- LOS: Rigid body motions of optical components on their mounts causes beam-shear - this is mostly low-order.

- Inertial: Shape change of primary or secondary mirror reacting against its mount due to mechanical accelerations.

- Thermal: Shape changes of telescope structure or individual optical components due to thermal environment. 


\section{Wavefront In-Stability: Inertial}

Inertial WFE is caused by the Primary Mirror reacting against its mount (i.e. rocking or bouncing) in response to accelerations (i.e. from the microthrusters).
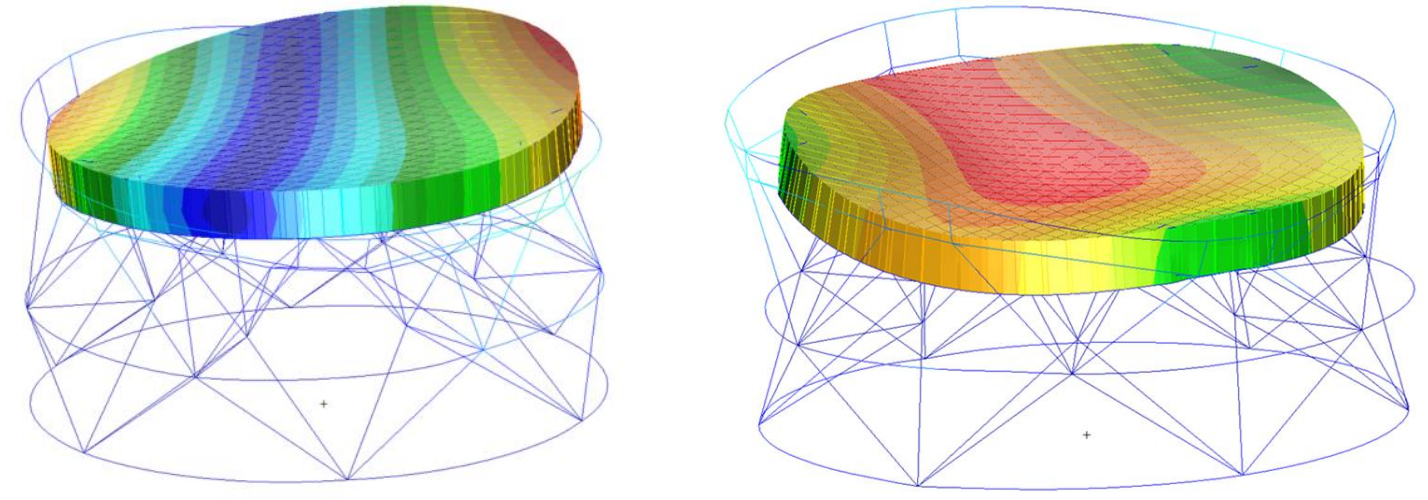

To minimize Inertial WFE:

- Design the PM Substrate to be as stiff as possible

- Consider the Mount stiffness and location.

NOTE: Inertial WFE is not caused by resonant motion. 


\section{Primary Mirror Assembly}

Dozens of Zerodur ${ }^{\circledR}$ and $\mathrm{ULE}^{\circledR}$ mirror designs were considered.

Baseline Zerodur ${ }^{\circledR}$ mirror design balances mass and stiffness.

- Substrate has a flat-back geometry with a $42 \mathrm{~cm}$ edge thickness and mass of approximately $1400 \mathrm{~kg}$.

- The mirror's free-free first mode frequency is $88 \mathrm{~Hz}$. And, its mounted first mode frequency is $70 \mathrm{~Hz}$.

- The mirror is locally stiffened to minimize gravity sag.

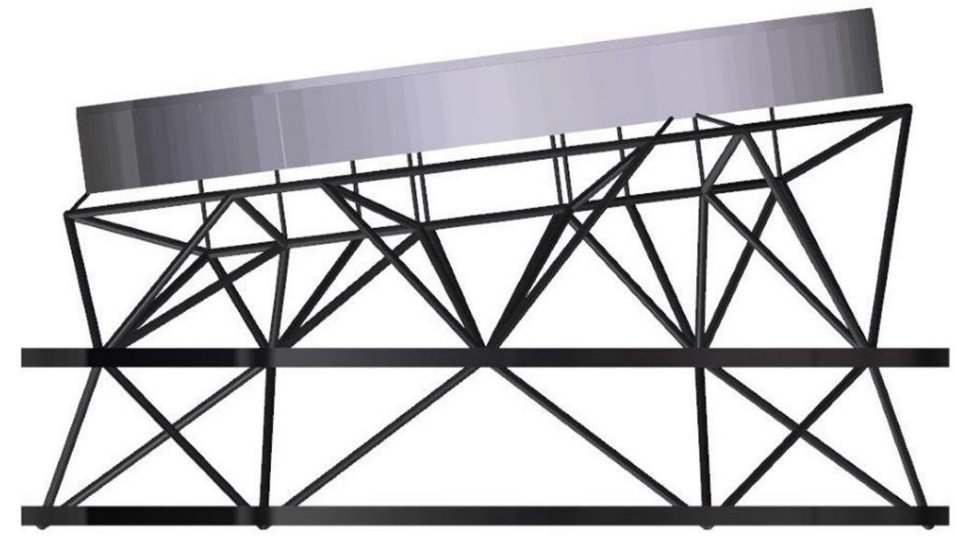

$$
\mathrm{X} \text {-axis }=18.6 \mu \mathrm{m} \text { RMS } \quad \text { Y-axis }=18.4 \mu \mathrm{m} \text { RMS }
$$




\section{Wavefront In-Stability: Thermal}

Thermal WFE instability occurs when the primary mirror's bulk temperature or temperature gradient changes.

If the mirror's coefficient of thermal expansion (CTE) is completely homogeneous and constant, then a bulk temperature should only result is a defocus error.

But any inhomogeneity in the mirror's CTE will result in a temperature dependent WFE.

Additionally, because CTE is itself temperature dependent, any change in the mirror's thermal gradient will also result in a WFE.

The best mitigation strategy is to actively control the mirror's thermal stability. 


\section{PM Thermal Stability}

Thermal WFE stability depends on the primary mirror's thermal sensitivity and the thermal system's controllability.

Rate at which the PM's RMS WFE changes depends on CTE, mass and specific heat $\left(c_{p}\right)$ :

$$
\frac{\delta S F E}{\delta t} \sim \frac{C T E}{M c_{p}}
$$

The larger a mirror's mass and smaller it's CTE, the smaller and slower its thermal response.

Thus want a zero CTE material. Also want zero CTE homogeneity

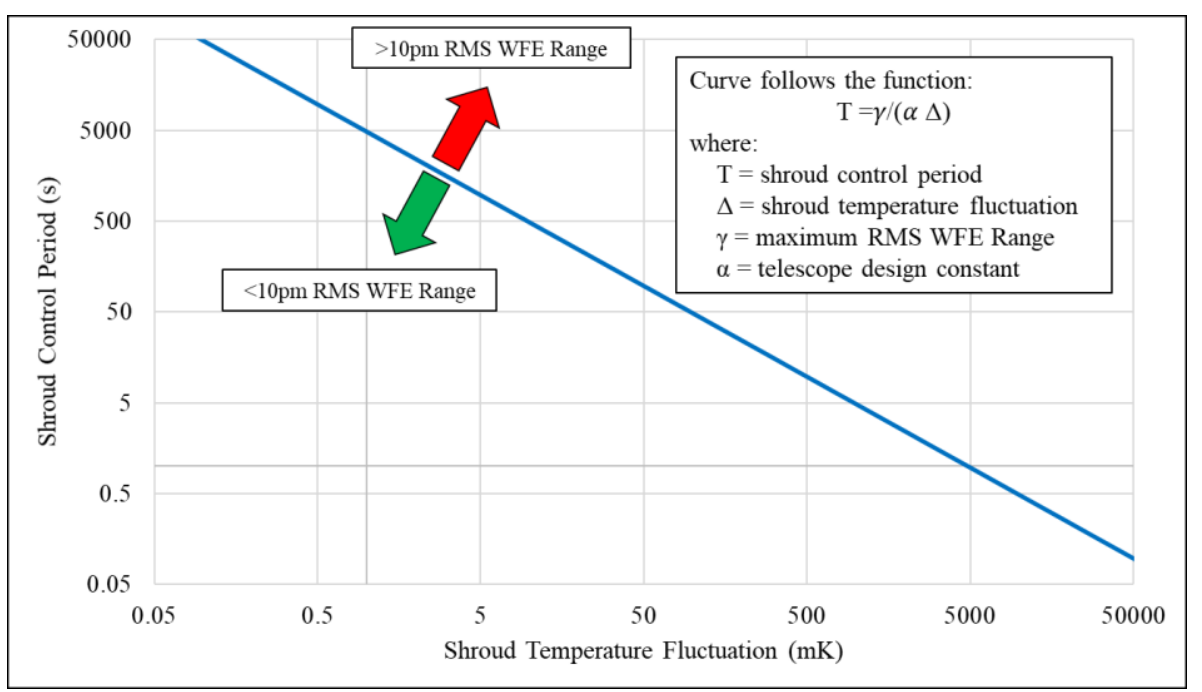




\section{Wavefront In-Stability: Thermal}

CTE homogeneity causes WFE as a function of thermal variation.

Again, this WFE is minimized by thermal control. BUT, the required control precision is proportional to CTE homogeneity.

The more homogeneous, the less precise the required control.

AMTD tested a $1.2 \mathrm{~m}$ Zerodur mirror and determined that its CTE homogeneity is approx. $+/-5 \mathrm{ppb} / \mathrm{K}$.

This mirror would meet WFE stability with $\sim 2 \mathrm{mK}$ thermal stability.

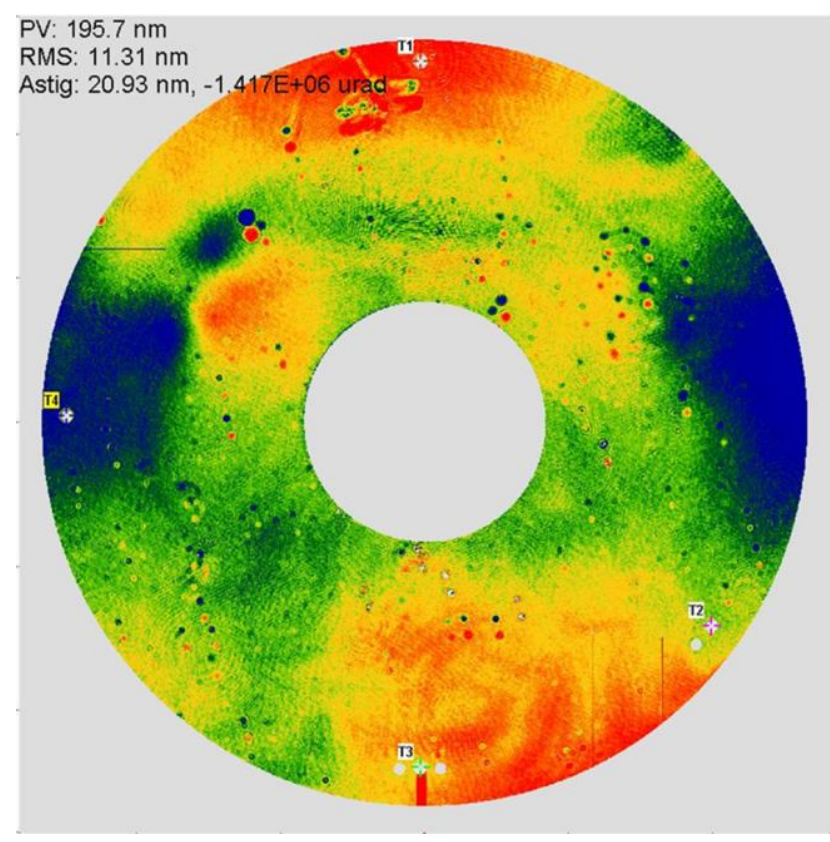




\title{
HabEx Baseline Primary Mirror
}

\author{
TRL Assessment
}




\section{Technology Readiness Level (TRL)}

NASA requires that the technology to manufacture and test the HabEx primary mirror must be:

- TRL-5 before start of Phase A

- TRL-6 before by PDR \& start of Phase C.

\begin{tabular}{|c|l|}
\hline TRL & \multicolumn{1}{|c|}{ Abridged definition } \\
\hline 1 & Basic principles observed and reported \\
\hline 2 & Concept and/or application formulated \\
\hline 3 & $\begin{array}{l}\text { Proof of concept hardware or model validation; critical } \\
\text { properties demonstrated }\end{array}$ \\
\hline 4 & $\begin{array}{l}\text { Low-fidelity component or breadboard in lab } \\
\text { demonstrates functionality and validates models that } \\
\text { predicts performance in relevant environment }\end{array}$ \\
\hline 5 & $\begin{array}{l}\text { Medium fidelity component or breadboard demonstrate } \\
\text { overall performance in relevant environment }\end{array}$ \\
\hline 6 & $\begin{array}{l}\text { High fidelity system/subsystem demonstrates critical } \\
\text { performance in operational environment; scaling is } \\
\text { understood }\end{array}$ \\
\hline 7 & $\begin{array}{l}\text { High fidelity engineering unit demonstrates } \\
\text { performance in operational environment }\end{array}$ \\
\hline 8 & System is flight qualified \\
\hline 9 & System flight performance successful \\
\hline
\end{tabular}




\section{TRL Assessment}

\section{HabEx assesses that the technology to manufacture a 4-m class flight mirror is currently TRL-4 because of 3 key technologies:}

- Ability to Certify that Zerodur Blank has CTE Homogeneity

- Ability to Certify that Mirror has Wavefront Error

- Ability to Certify that Mirror will achieve Wavefront Stability

\begin{tabular}{|c|c|c|c|}
\hline \multicolumn{4}{|c|}{ Large Nitror Fabrication } \\
\hline Technology & Need & State of the Art & TRL \\
\hline Mirror Substrate Diameter & 4.04 meter & Schott Corp manufactures blanks that are $4.2 \mathrm{~m}$ diameter $\mathrm{x} 420 \mathrm{~mm}$ thick & 6 \\
\hline \multicolumn{4}{|l|}{ Mirror Substrate CTE } \\
\hline - $\quad$ Bulk CTE & 0 at $270 \mathrm{~K}$ & Schott Corp can tune CTE to be 0 at a specific temperature. & 6 \\
\hline CTE Homogeneity & $\begin{array}{l}<+/-5 \mathrm{ppb} / \mathrm{K} \text { over } 100 \times 100 \\
\text { spatial sampling }\end{array}$ & Schott Corp demonstrated $<+/-3 \mathrm{ppb} / \mathrm{K}$ over limited spatial sampling on DKIST & 4 \\
\hline Substrate Machining & $\begin{array}{l}3-4 \mathrm{~mm} \text { ribs, } 14 \mathrm{~mm} \text { facesheet, } \\
\text { and pocket depth of } 290 \mathrm{~mm} \text { for } \\
400 \mathrm{~mm} \text { thick blank }\end{array}$ & $\begin{array}{l}\text { Schott Corp demonstrated computer-controlled-machine lightweighting to pocket } \\
\text { depth of } 340 \mathrm{~mm}, 4 \mathrm{~mm} \text { rib thickness on E-ELT M5 and } 240 \mathrm{~mm} \text { deep/2 } \mathrm{mm} \\
\text { thick rib on Schott } 700 \mathrm{~mm} \text { diameter test unit }\end{array}$ & 6 \\
\hline Areal Density & $110 \mathrm{~kg} \cdot \mathrm{m}^{2}$ & $\begin{array}{l}\text { State-of-the-practice lightweighting has made large glass mirrors with aerial } \\
\text { density of } 70 \mathrm{~kg} / \mathrm{m}^{2}\end{array}$ & 6 \\
\hline First Mode Frequency & $\geq 60 \mathrm{~Hz}$ & $\begin{array}{l}\text { By design, if the baseline Zerodur }{ }^{\circledR} \text { mirror substrate can be machined to its } \\
\text { specified dimensions using demonstrated Schott Corp machining capability, it } \\
\text { will achieve the required first mode frequency. Also, sub-scale WFIRST } 2.4-\mathrm{m} \\
\text { Primary Mirror has } \sim 200 \mathrm{~Hz} \text { first mode. }\end{array}$ & 6 \\
\hline Wavefront Error & $\begin{array}{l}0-7 \text { cy/D: } 6.9 \mathrm{~nm} \text { RMS } \\
7-100 \mathrm{cy} / \mathrm{D}: 6.0 \mathrm{~nm} \text { RMS } \\
>100 \mathrm{cy} / \mathrm{D}: 0.8 \mathrm{~nm} \text { RMS }\end{array}$ & Demonstrated on sub-scale WFIRST 2.4-m Primary Mirror & 4 \\
\hline Wavefront Stability & 1 to $100 \mathrm{pm} \mathrm{rms}$ & $\begin{array}{l}\text { By design, baseline Zerodur }{ }^{\circledR} \text { mirror will achieve required wavefront stability } \\
\text { with active zonal thermal control stability of }<5-\mathrm{mK} \text {. Sub-scale active thermal } \\
\text { control has been demonstrated by Harris Corp to TRL- } 9 \text { on } 1.1-\mathrm{m} \text { Spaceview }{ }^{\mathrm{TM}}\end{array}$ & 4 \\
\hline
\end{tabular}

\section{These can be matured to TRL-5 by 2024 with modest investment.}

Proc. SPIE 11116, Astronomical Optics: Design, Manufacture and Test of Space and Ground Systems, August 2019 


\section{Mirror Material}

A key metric for selecting the HabEx primary mirror material is coefficient of thermal expansion (CTE).

- CTE and CTE homogeneity are important because they determine how the mirror's shape deforms as a function of bulk temperature or thermal gradient changes.

- Such deformations impacts the telescopes ability to meet its diffraction limited performance and wavefront stability specifications.

Typical approaches for mitigating this risk are

- Select a material with zero CTE and extreme homogeneity.

- Measure the mirror's shape change between its manufacture and operational temperatures and 'cryo-null figure' the mirror

- Actively control the mirror's on-orbit shape or its thermal environment. 


\section{Mirror Material}

Both Zerodur ${ }^{\circledR}$ and ULE $^{\circledR}$ are TRL-9 with multiple mirrors flying. Both Schott and Corning can tailor their material's zero CTE temperature.
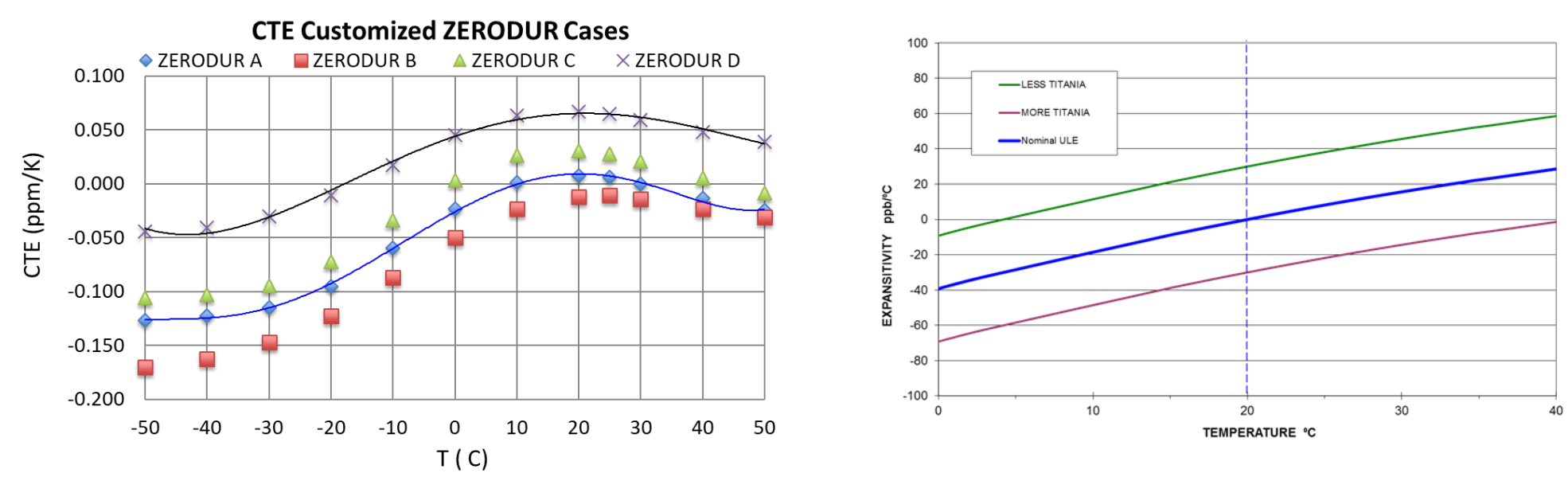

And both claim similar CTE homogeneity (i.e. +/- 5 ppb).

Thus, a mirror manufactured from either material should have similar thermal performance. 


\section{Mirror Material}

A significant difference between $\mathrm{ULE}^{\circledR}$ and $\mathrm{Zerodur}^{\circledR}$ is the design architecture enabled by each.

As a glass, $\mathrm{ULE}^{\circledR}$ can be assembled to enable closed-back mirror architectures. Such mirrors are stiffer.

As a ceramic, Zerodur ${ }^{\circledR}$ must be machined from a single blank. Thus, Zerodur ${ }^{\circledR}$ mirrors required an open-back architecture.

However, because Zerodur ${ }^{\circledR}$ mirrors are machined from a single boule, they may have a smoother and more homogeneous CTE. 


\section{Primary Mirror Material Selection}

Zerodur $^{\circledR}$ was selected as the baseline HabEx primary mirror material because Schott has demonstrated a routine ability to manufacture 4-m class mirror blanks.

This demonstrated capability enables HabEx to assess the ability to make 4-m class mirror blanks to be TRL-6.
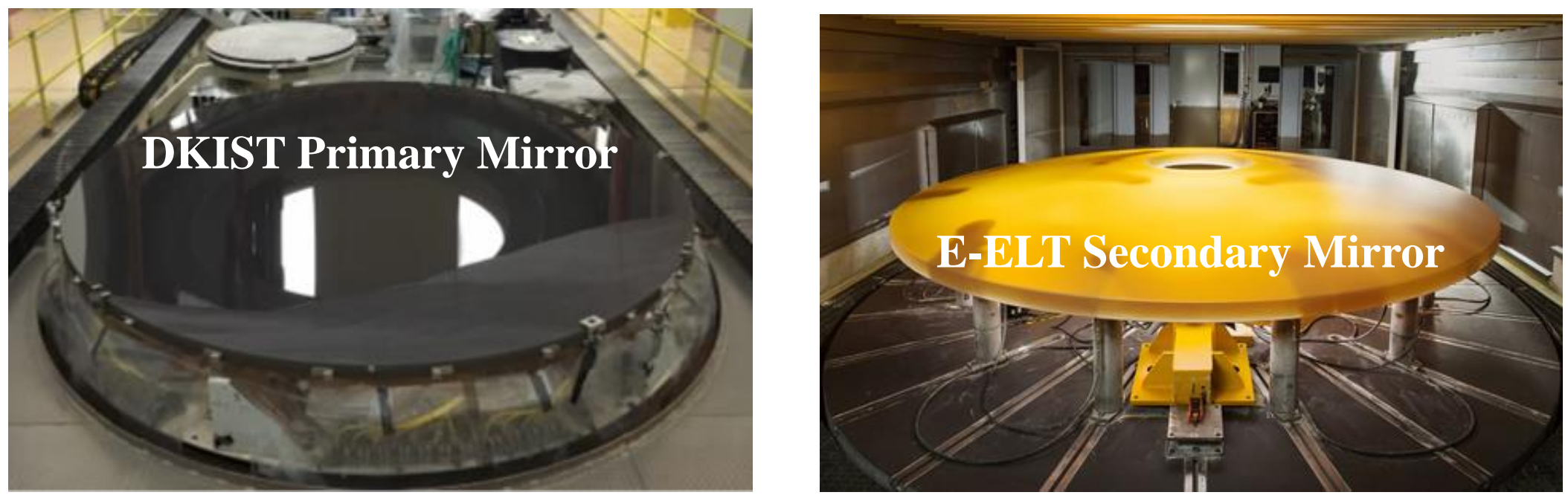


\section{Substrate Machining}

Schott Corp has the infrastructure (including a 5-m 5 axis CNC Machine) to machine deep core structures as large as 4.5 -meters.
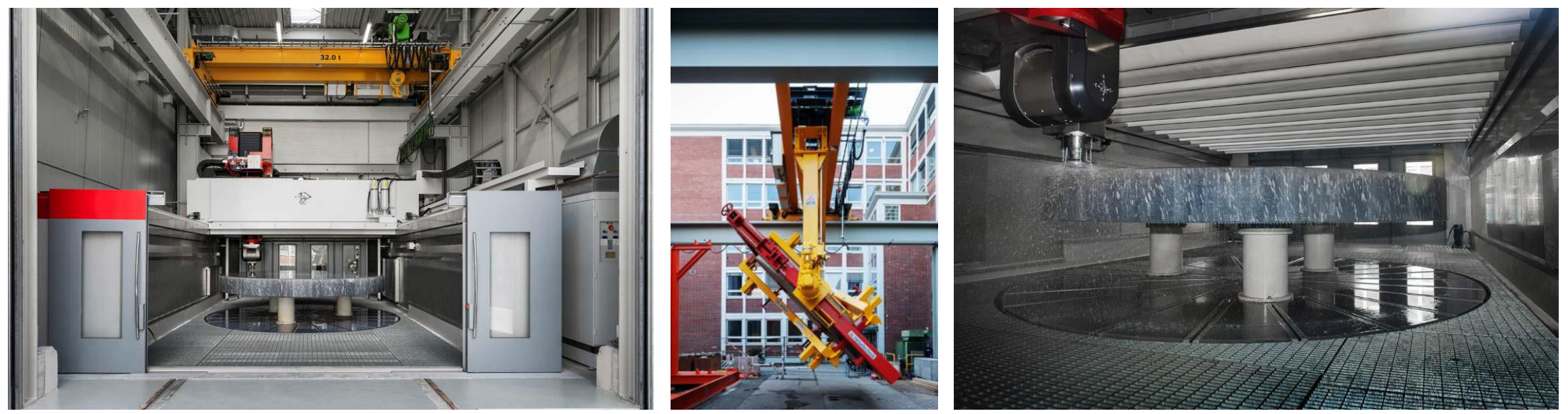

Machining high-fidelity sub-scale mirrors has capability at TRL-6

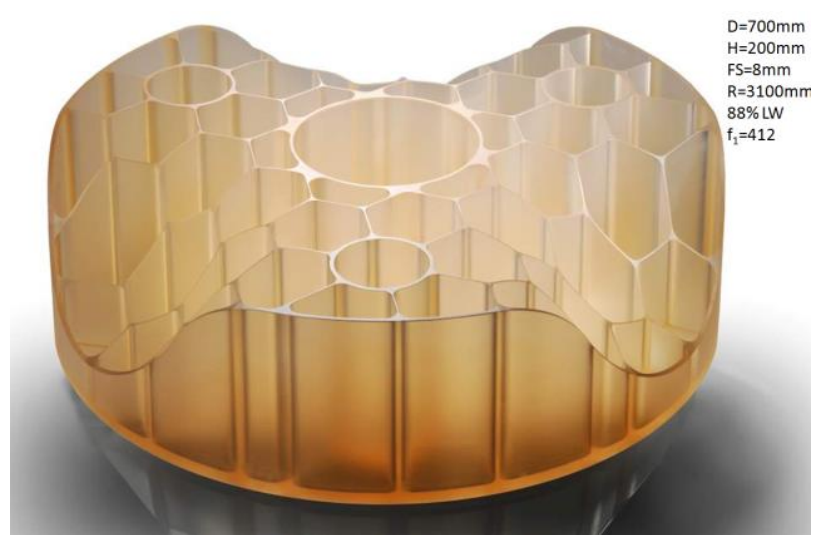

0.7-m diameter, $200 \mathrm{~mm}$ thick mirror with 2 mm machined walls.

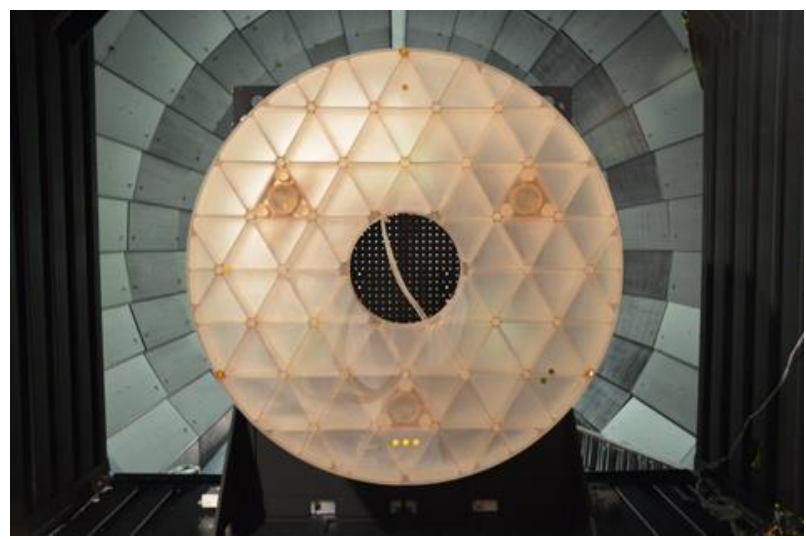

1.2-m diameter, 125-mm thick mirror with

$2 \mathrm{~mm}$ machined walls. 


\section{Polishing Infrastructure}

Multiple organizations have existing infrastructure to grind and polish 4-m class substrates into space mirrors, including: Collins Aerospace, L3/Brashears, Harris Cor., Arizona Optical Systems, University of Arizona, and REOSC.

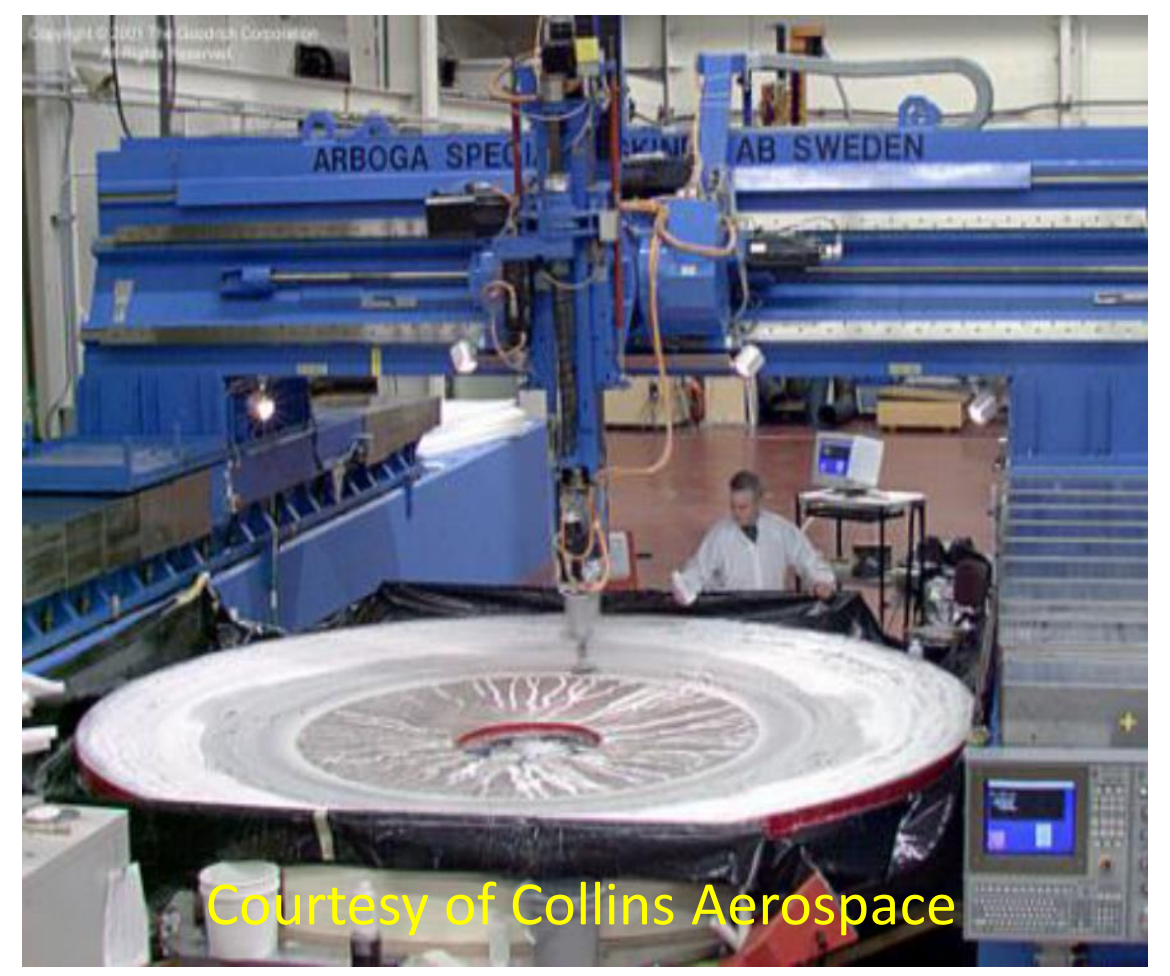




\section{Surface Figure Error}

Polishing mirrors to required specification at 2.4-m is TRL-9. HabEx specification is same as WFIRST's current surface.

As will be discussed in the next section, the only risk is the ability to quantify and back-out gravity induced self-weight deflection to $\sim 2 \mathrm{~nm}$ rms over a $100 \times 100$ spatial sampling is assessed to be at TRL-4. 


\section{Wavefront Stability}

As will be discussed in the next section, Schott's ability to provide HabEx PM with <+/-5 ppb/K CTE homogeneity is assessed at TRL-4 because they do not have a non-destructive process for validating CTE homogeneity on a 4-m class mirror over 100 x 100 spatial sampling. 


\section{Thermal Control System TRL}

Baseline HabEx thermal control system is assessed to be TRL-4.

System is scale-up of TRL-9 system built by Harris Corp.

- Harris is flying $0.7 \& 1.1-\mathrm{m}$ systems on its Spaceview ${ }^{\mathrm{TM}}$ telescopes.

- Harris built 1.5-m system built with 37 thermal control zones for MSFC Predictive Thermal Control Study.
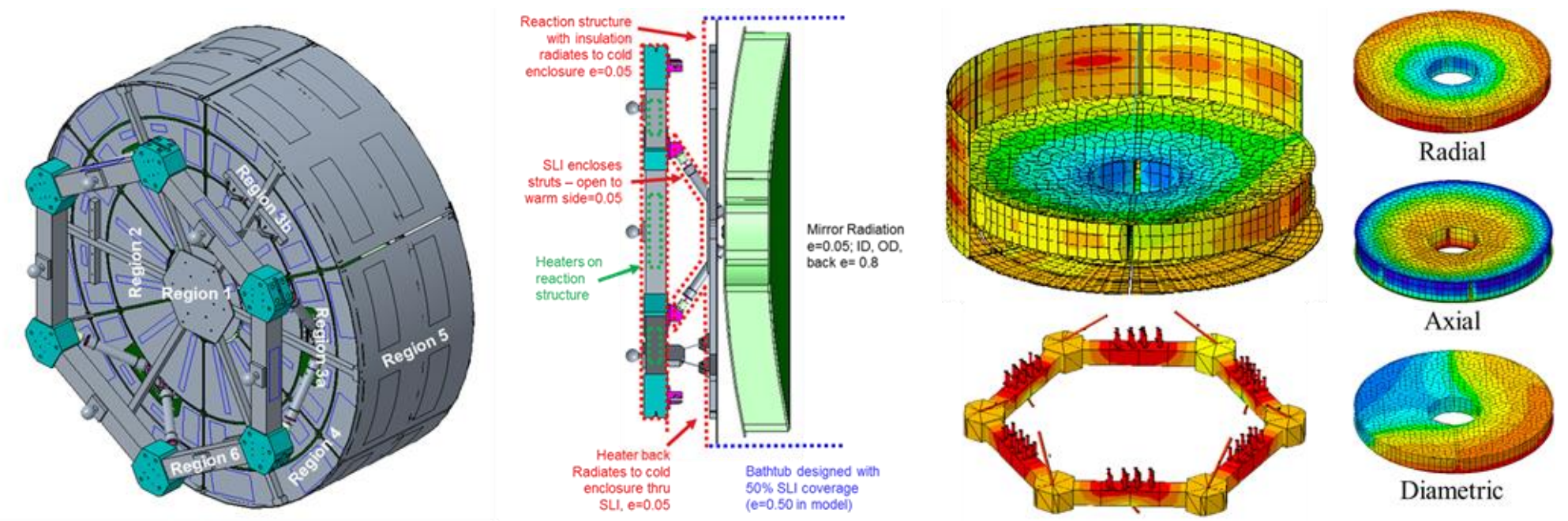

Analysis indicates that, because of PM thermal mass, system with $0.5-\mathrm{Hz}, 50-\mathrm{mK}$ sensors will keep PM stable to $\sim 1-\mathrm{mK}$. 


\title{
HabEx Baseline Primary Mirror
}

\author{
Technology Maturation
}




\section{CTE Homogeneity State of Practice}

\section{Schott Corp has a dilatometer process that can measured CTE of test samples with a reproducibility of $\sim+/-1 \mathrm{ppb} / \mathrm{K}$.}

Since 20010, Schott has produced seven 4-m mirror substrates with CTE homogeneity $<10 \mathrm{ppb} / \mathrm{K}$. And one with $3 \mathrm{ppb} / \mathrm{K}$.

\begin{tabular}{|c|c|c|c|c|c|c|}
\hline & Dimension & $\begin{array}{c}\text { Number of } \\
\text { Samples }\end{array}$ & $\begin{array}{c}\text { CTE }\left(\mathbf{0}^{\circ} ; \mathbf{5 0}^{\circ}\right) \text { absolute value } \\
{[\mathbf{p p b} / \mathbf{K}]}\end{array}$ & $\begin{array}{c}\text { CTE }\left(\mathbf{0}^{\circ} ; \mathbf{5 0}^{\circ}\right) \text { homogeneity } \\
{[\mathbf{p p b} / \mathbf{K}]}\end{array}$ \\
\hline Year & {$[\mathbf{m m}]$} & $\#$ & Specification & Achieved & Specification & Achieved \\
\hline 2003 & $4100 \times 171$ & 18 & $+/-50$ & 66 & 20 & $18^{1}$ \\
\hline 2005 & $3610 \times 370$ & 12 & $+/-100$ & 80 & 30 & $25^{1}$ \\
\hline 2009 & $3700 \times 163$ & 36 & $+/-150$ & 54 & 40 & 9 \\
\hline 2010 & $3400 \times 180$ & 12 & $+/-100$ & 42 & 30 & 5 \\
\hline 2012 & $4250 \times 350$ & 16 & $+/-30$ & 60 & 40 & 5 \\
\hline 2014 & $4250 \times 350$ & 16 & $+/-30$ & 0 & 40 & 3 \\
\hline 2016 & $4060 \times 103$ & 16 & $+/-50$ & 36 & 20 & 7 \\
\hline 2016 & $4000 \times 100$ & 12 & $+/-150$ & 15 & 20 & 4 \\
\hline 2019 & $4250 \times 100$ & 20 & $+/-20^{*}$ & $-9^{*}$ & $20^{*}$ & $8^{*}$ \\
\hline
\end{tabular}

R. Jedamzik, T. Westerhoff, "Homogeneity of the coefficient of linear thermal expansion of ZERODUR ${ }^{\circledR}$ : A Review of a decade of evaluations" Proc. SPIE Vol. 10401, (2017)

Westerhoff, Thomas, and Tony Hull, "Production of 4 m diameter Zerodur ${ }^{\circledR}$ mirror substrates", HabEx White Paper Contribution, 2018. 


\section{CTE Homogeneity State of Practice}

But, because acquiring CTE samples is destructive, data sampling is limited to perimeters and holes.
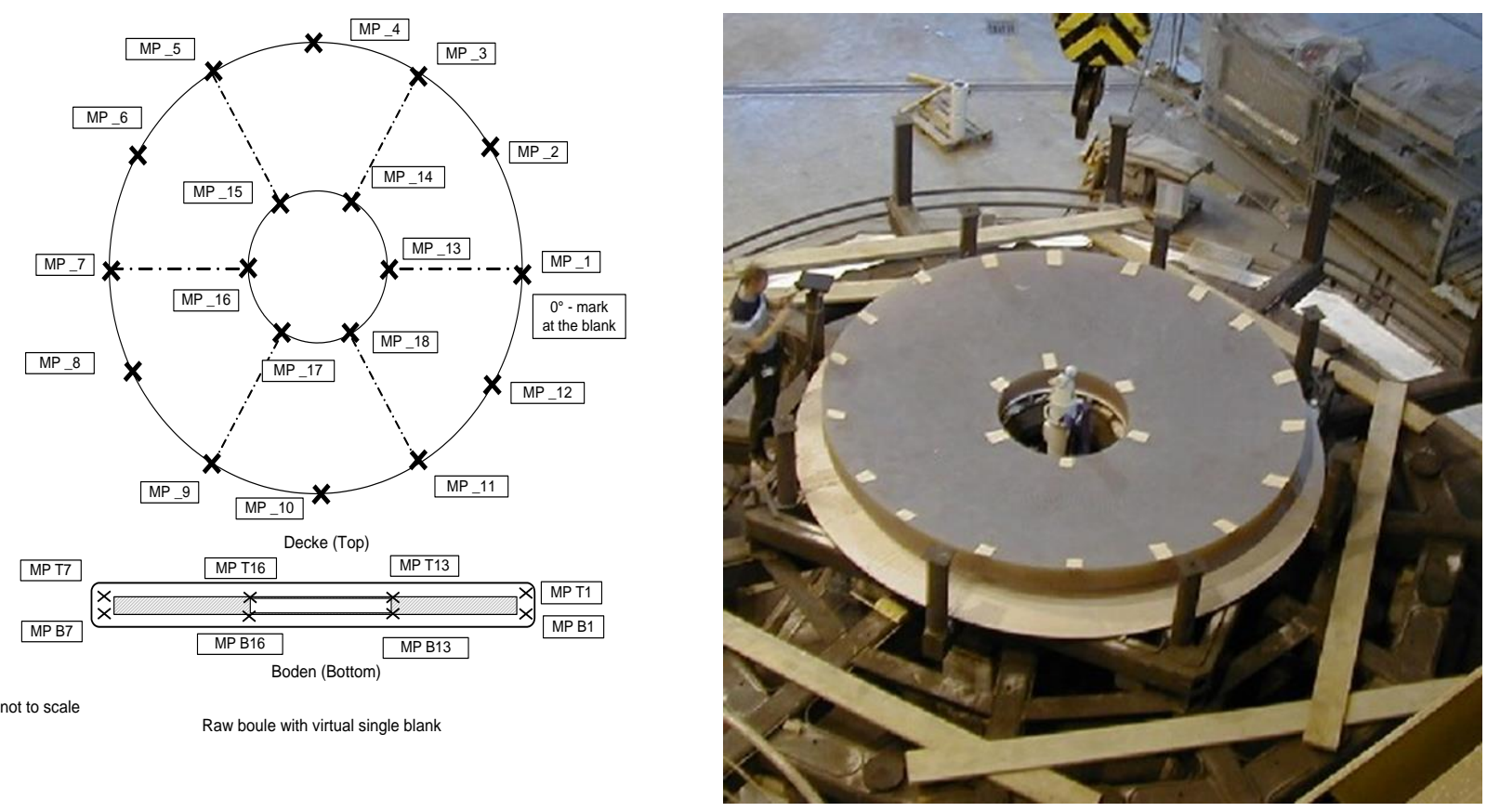

Thus, HabEx requires a 'validated' non-destructive process to certify CTE homogeneity of the primary mirror blank over at least a $100 \times 100$ spatial sampling as part of the primary mirror blank acceptance process. Tony Hull Private Communication 


\section{CTE Homogeneity Characterization Process}

Proposed Flight CTE Homogeneity Characterization Process:

- Cast two 4-m Boules and machine them into Blanks

- Polish best fit radius sphere into each solid Blank

- Measured cryo-deformation of each Blank over $100 \mathrm{~K}$ range.

- Select Blank with smallest cryo-deformation for Flight PM

- Use CTE data to adjust PM lightweighting Design.

\section{Proposed Validation of Process:}

- Procure two sub-scale blanks with polished spherical surfaces

- Measure cryo-deformation of each blank

- Cut up one blank into 100 x 100 CTE dilatometer samples

- Measure CTE of each sample with uncertainty $1 \mathrm{ppb} / \mathrm{K}$

- Correlate CTE samples with Cryo-Deformation Map

- Machine $2^{\text {nd }}$ blank into lightweight mirror

- Re-measure cryo-deformation of $2^{\text {nd }}$ mirror to determine if lightweight machining changes its cryo performance. 


\section{CTE Homogeneity Characterization}

Schott has mapped CTE homogeneity of meter class blanks, but this mapping has not been correlated with Cryo-Deformation.

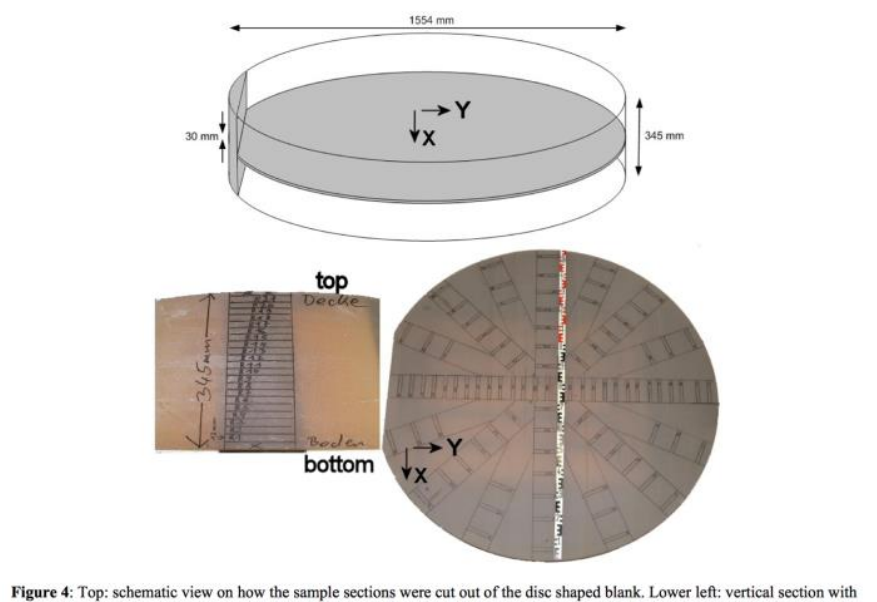

sample markings, lower right: horizontal section with sample markings.

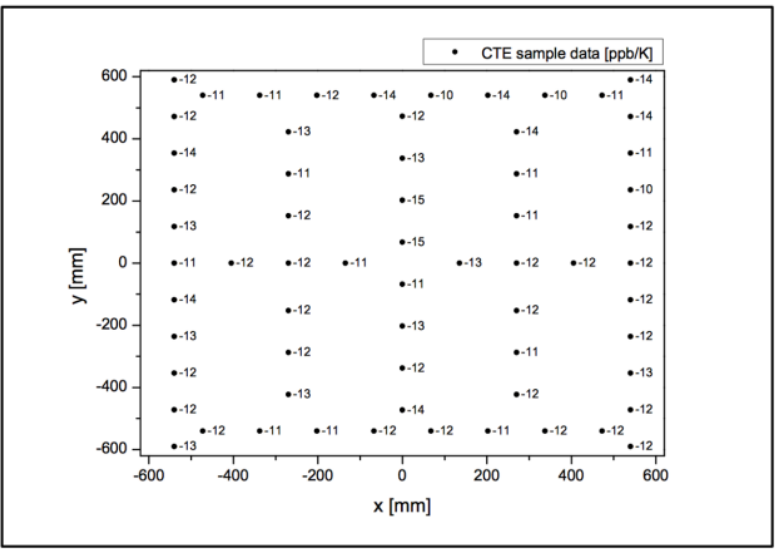

Sample distribution and measured CTE values in ppb / K within the $1200 \mathrm{~mm} \times 1200 \mathrm{~mm}$ plate

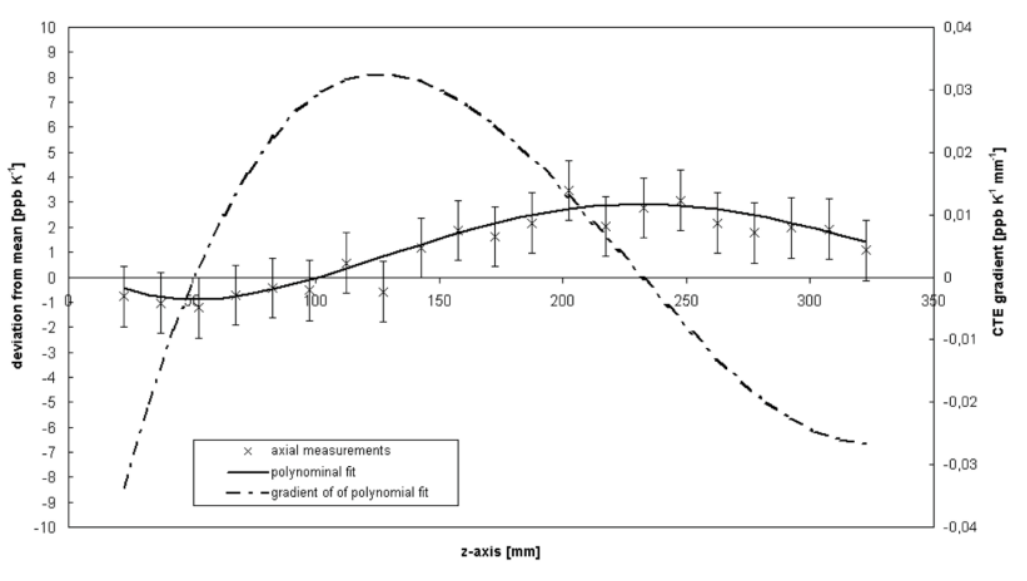

Figure 9: Variation of the CTE deviations from the mean value and gradient in axial direction

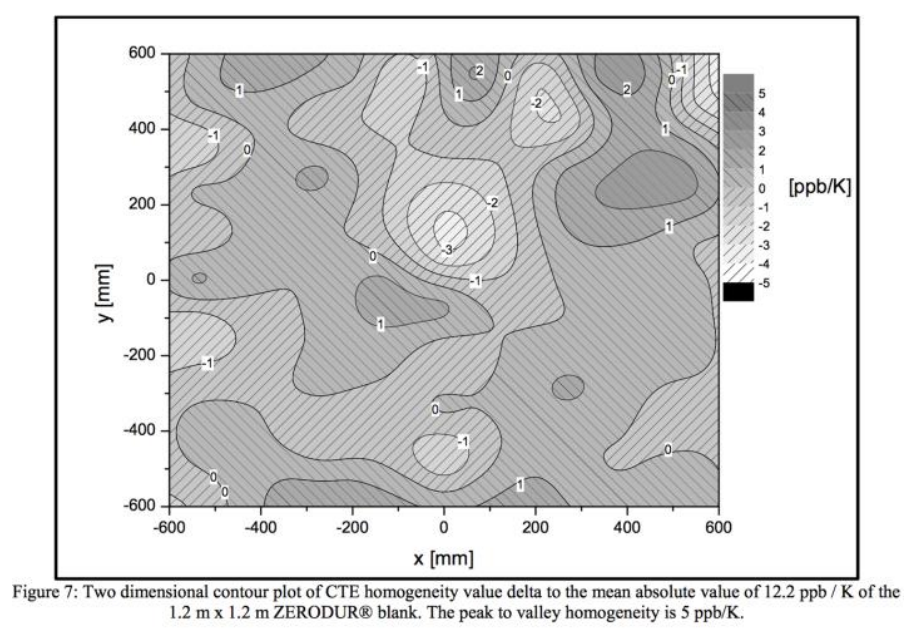

Proc. SPIE 11116, Astronomical Optics: Design, Manufacture and Test of Space and Ground Systems, August 2019 


\section{Gravity Deformation State of Practice}

Gravity-sag characterization and mitigation have been studied extensively since the 1960 s.

Mitigation approaches:

- Minimize Gravity Sag by making the mirror as stiff as possible and optimizing its mounting

- Off-load Gravity during fabrication and test

- Analytically removed Gravity Sag during test

- Actively correct mirror shape on-orbit 


\section{Minimize Gravity Sag}

\section{Design the mirror to be as stiff as possible}

$$
1 \text { G Gravity Sag } \sim C_{S P}\left(\frac{D^{4}}{t^{3}}\right) \rho_{A D} \sim 1 /(2 \pi f)^{2}
$$

\section{And optimize its mounting}

\begin{tabular}{|l|c|c|}
\hline \multicolumn{1}{|c|}{ Support Constant } & $\mathbf{C}_{\mathrm{SP}}$ & $\begin{array}{c}\text { Factor of Reduced Deflection } \\
\text { Compared to 3-Pt Support }\end{array}$ \\
\hline Ring at 68\% of Diameter & 0.028 & 11 \\
\hline 6 Points Equal Spaced at 68.1\% of Diameter & 0.041 & 8 \\
\hline Edge Clamped & 0.187 & 1.5 \\
\hline 3 Points, Equal Spaced at 64.5\% of Diameter & 0.316 & - \\
\hline 3 Points, Equal Spaced at 66.7\% of Diameter & 0.323 & $\sim 1$ \\
\hline 3 Points, Equal Spaced at 70.7\% of Diameter & 0.359 & 0.9 \\
\hline Edge Simply Supported & 0.828 & $1 / 3$ \\
\hline Continuous Support along the Diameter & 0.943 & $1 / 3$ \\
\hline “Central Support” (Mushroom or Stalk Mount; $r$ radius of stalk) & 1.206 & $1 / 4$ \\
\hline 3 Points Equal Spaced at Edge & 1.356 & $1 / 4$ \\
\hline
\end{tabular}

Yoder, Paul and Danial Vukobratovich, Opto-Mechanical Systems Design, Fourth Edition, Two Volume Set, CRC, 2015

\section{Yields Baseline Zerodur ${ }^{\circledR}$ mirror designed to minimize gravity sag}

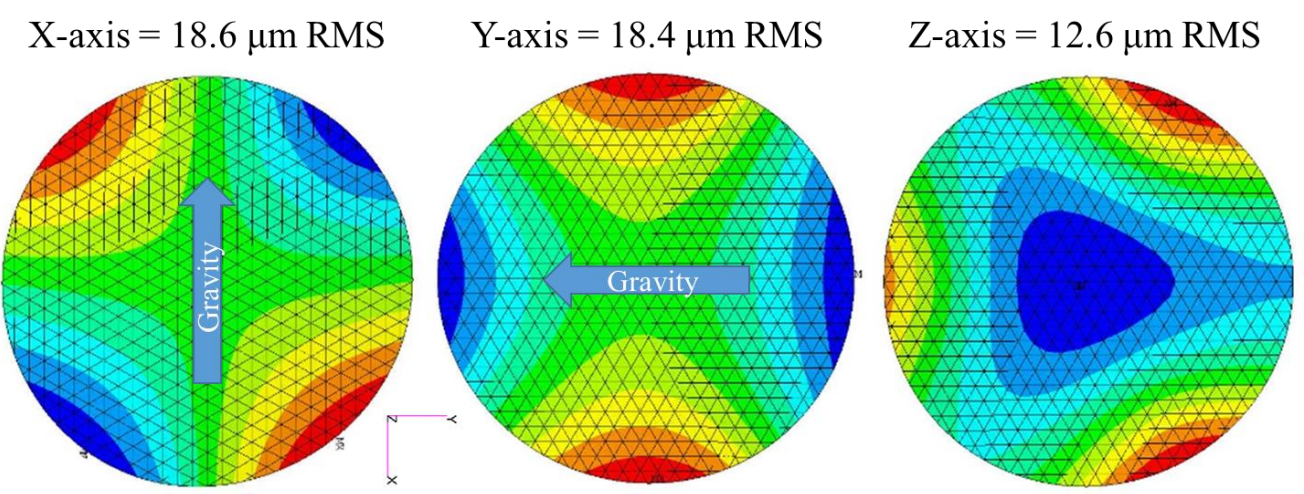




\section{Gravity Off-Loading}

Gravity off-loading is typically done via:

- Multipoint Mount

- Air Bag Support

Hubble PM's 7.6 micrometer G-sag was characterized to an accuracy of $1.4 \mathrm{~nm}$ rms using a 135 point metrology mount.

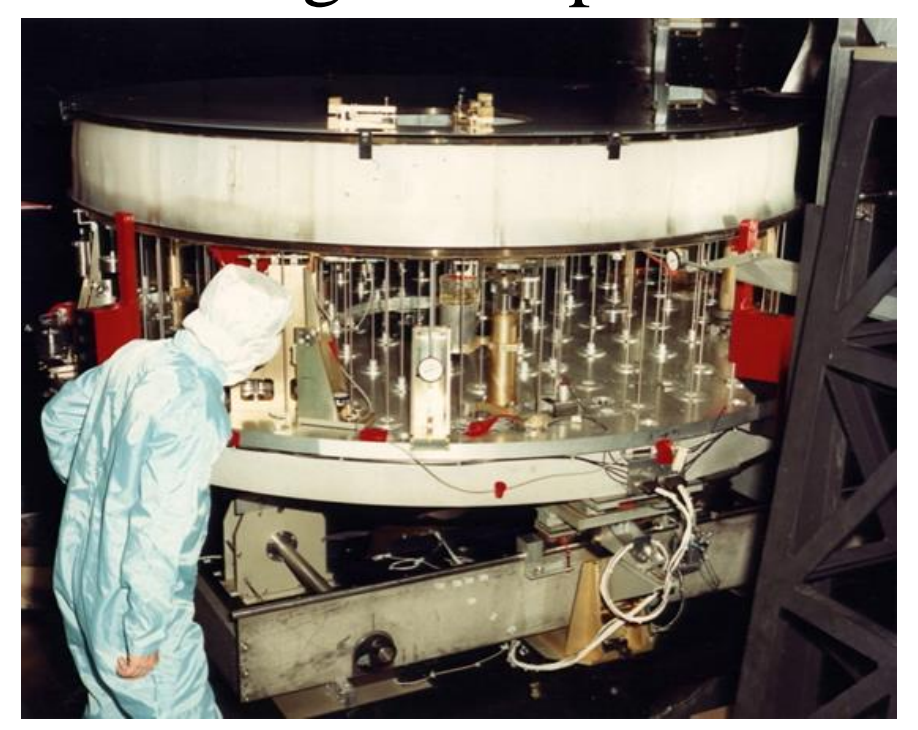

Yoder, Paul and Danial Vukobratovich, Opto-Mechanical Systems Design, Fourth Edition, Two Volume Set, CRC, 2015 ISBN-10: 1439839778

https://www.hexagonkh9.com/blog/2019/1/19/hexagon-looked-at-the-earth-the-hubble-looked-at-the-stars 


\section{Analytical Removal}

Gravity Sag can be estimated via an orientation test:

- Face-up/Face-down Test

- Horizontal Rotation Test

JWST segments were tested using rotation test to $<10 \mathrm{~nm}$ rms. 


\section{Kepler Gravity Sag}

Kepler primary mirror was tested using an air bag, a 108 point metrology mount, and a face-up/face-down orientation test.

Air bag was estimated to off-load gravity sag to $5.6 \mathrm{~nm} \mathrm{rms}$.

Difference between air bag \& multi-point mount was $16.4 \mathrm{~nm}$ rms

Difference between air bag \& face-up/face-down was $18.4 \mathrm{~nm}$ rms

Largest component of difference was spherical aberration.

By inference, difference between multipoint mount and up/down test should be $8.3 \mathrm{~nm}$ rms.

Zinn, John W., George W. Jones, "Kepler primary mirror assembly: FEA surface figure analyses and comparison to metrology,"

Proc. SPIE 6671, Optical Manufacturing and Testing VII, 667105 (14 September 2007) 


\section{Gravity Deformation Characterization Process}

Manufacturing a 4-m class $80-\mathrm{Hz} 400-\mathrm{nm}$ wavelength diffraction limited mirror requires a validated process to quantify the zerogravity shape of the mirror on its flight-mount.

Process capabilities:

- Dynamic range of $\sim 100$ micrometers PV

- Measurement uncertainty < 5 nanometers rms (goal of $<2 \mathrm{~nm} \mathrm{rms}$ )

- Spatial sampling > $100 \times 100$ (dark-hole outer working angle)

To achieve TRL-6, don't need demonstrate on a 4-m mirror.

Only necessary to demonstrate on a mirror with a 'representative' first mode stiffness, i.e. an $80-\mathrm{Hz}$ mirror at $1.5-\mathrm{m}$ or $2-\mathrm{m}$ that has the same gravity sag as an $80-\mathrm{Hz} 4-\mathrm{m}$ mirror.

AND, mirror should be a sphere - to eliminate alignment issues. 


\section{Gravity Deformation Characterization Process}

\section{Proposed Gravity Deformation Characterization Process}

- Analytically determine 0-G surface using orientation tests.

- Predict horizontal and vertical gravity-sag of a test-article mirror assembly using a high-fidelity FEM and create vertical and horizontal CGHs.

- Quantify gravity-sag of test-article mirror assembly using N-rotation and face-up/face-down methods.

- Horizontal test - astigmatic gravity sag may be significant. So, use a CGH that does not rotate to compensate. N-Rotation test will yield difference between CGH and actual G-sag.

- Up/down test - use 2 CGHs to compensate. When placed together, 2 CGHs should produce no error when testing a calibration sphere.

- Achieve 0-G surface on a multipoint fabrication/metrology mount.

- Generate a spatial stiffness map of mirror

- Mount mirror to a multipoint fabrication/metrology mount

- 'Poke' mirror at support point and measure mirror's influence function.

- Solve for mirror's local stiffness at each point.

- Calculate up-force needed to off-load gravity at each point.

- Correlate 0-G surface from orientation tests with multi-point mount. 


\section{Conclusions}

- HabEx requires a 4-m primary mirror with a $0-\mathrm{G}$ surface:

Total SFE

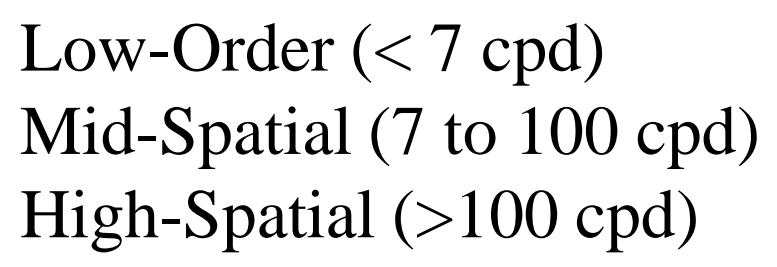

WFE Stability
$<8 \mathrm{~nm} \mathrm{rms}$

\section{$<7 \mathrm{~nm} \mathrm{rms}$}

$<6 \mathrm{~nm} \mathrm{rms}$

$<0.8 \mathrm{~nm} \mathrm{rms}$

$<1$ to 200 pm rms.

- Technology to manufacture and certify mirror is assessed to be TRL-6 except for two TRL-4 items:

○ Non-destructive process to quantify CTE homogeneity of a 4-m black over spatial sampling of at least $100 \times 100$ to $<+/-5 \mathrm{ppb} / \mathrm{K}$.

- Process to quantify self-weight gravity deflection to $\sim 2 \mathrm{~nm}$ rms over a $100 \times 100$ spatial sampling.

- Roadmap is defined to mature these Technologies to TRL-6. 